\title{
Thick-skinned 3-manifolds
}

\author{
Richard P. Kent IV and Yair N. Minsky
}

June 30, 2014

\begin{abstract}
We show that if the totally geodesic boundary of a compact hyperbolic 3manifold $M$ has a collar of depth $d \gg 0$, then the diameter of the skinning map of $M$ is no more than $A e^{-d}$ for some $A$ depending only on the genus and injectivity radius of $\partial M$.
\end{abstract}

Given a discrete group $G$, we equip $\operatorname{Hom}\left(G, \mathrm{PSL}_{2}(\mathbb{C})\right)$ with the compact-open topology. This induces a topology on the $\operatorname{space} \operatorname{Hom}\left(G, \mathrm{PSL}_{2}(\mathbb{C})\right) / \mathrm{PSL}_{2}(\mathbb{C})$ of conjugacy classes of representations called the algebraic topology. If $N$ is a connected 3-manifold, we let

$$
\operatorname{AH}(N) \subset \operatorname{Hom}\left(\pi_{1}(N), \mathrm{PSL}_{2}(\mathbb{C})\right) / \mathrm{PSL}_{2}(\mathbb{C})
$$

be the subset of conjugacy classes of discrete and faithful representations with the subspace topology. Each such conjugacy class corresponds to a hyperbolic structure on a 3-manifold homotopy equivalent to $N$. Let $\mathrm{AH}(N)^{\circ}$ be the interior of $\mathrm{AH}(N)$.

Let $S$ be a closed connected oriented surface of negative Euler characteristic.* By work of Marden [14] and Sullivan [23], the space $\mathrm{AH}(S \times \mathbb{R})^{\circ}$ equals the set $\mathrm{QF}(S)$ of convex cocompact, or quasifuchsian, hyperbolic structures on $S \times \mathbb{R}$. By the Simultaneous Uniformization Theorem [3], the space $\mathrm{QF}(S)$ is naturally homeomorphic to the product of Teichmüller spaces $\mathscr{T}(S) \times \mathscr{T}(\bar{S})$. If $(X, \bar{Y})$ is a point of $\mathscr{T}(S) \times \mathscr{T}(\bar{S})$, we let $\mathrm{qf}(X, \bar{Y})$ denote $S \times \mathbb{R}$ with the corresponding convex cocompact hyperbolic structure.

Let $M$ be a compact hyperbolic 3-manifold with totally geodesic boundary homeomorphic to $S$. A generalization of the Simultaneous Uniformization Theorem due to Ahlfors, Bers, Marden, and Sullivan (see [5] or [8]) tells us that the space $\mathrm{AH}(M)^{\circ}$ of convex cocompact hyperbolic metrics on $M^{\circ}$ is naturally homeomorphic to the Teichmüller space $\mathscr{T}(S)$. If $X$ is a point in $\mathscr{T}(S)$, we let $M^{X}$ denote $M^{\circ}$ equipped with the corresponding convex cocompact hyperbolic structure.

This research was partially supported by NSF grants DMS-1104871 and DMS-1005973. The authors acknowledge support from U.S. National Science Foundation grants DMS-1107452, 1107263, 1107367 "RNMS: GEometric structures And Representation varieties" (the GEAR Network).

*We assume that $S$ is connected for convenience. Defining the Teichmüller space of a disconnected surface to be the product of the Teichmüller spaces of the components, and adapting the proofs accordingly, we could dispense with this assumption. 
The inclusion $\partial M \rightarrow M$ induces a map $\mathrm{AH}(M)^{\circ} \rightarrow \mathrm{QF}(S)$. Identifying $\mathrm{AH}(M)^{\circ}$ with $\mathscr{T}(S)$ and $\mathrm{QF}(S)$ with $\mathscr{T}(S) \times \mathscr{T}(\bar{S})$, this map is given by $X \mapsto\left(X, \sigma_{M}(X)\right)$ for some function

$$
\sigma_{M}: \mathscr{T}(S) \rightarrow \mathscr{T}(\bar{S}) .
$$

The function $\sigma_{M}$ is Thurston's skinning map associated to $M$. This map is a key ingredient in Thurston's proof of Geometrization for Haken Manifolds [17, 20, 21, 11]. Thurston's Bounded Image Theorem [24, 12] states that the image of $\sigma_{M}$ is bounded, and we call the diameter of the image with respect to the Teichmüller metric the diameter of $\sigma_{M}$. In [12], the first author proved that if $\partial M$ has a large collar, then $\sigma_{M}$ carries a large ball to a set of very small diameter (Theorem 29 there). We greatly improve that theorem here.

We say that the totally geodesic boundary $\partial M$ in a hyperbolic 3-manifold $M$ has a collar of depth $d$ if the $d$-neighborhood of $\partial M$ is homeomorphic to $\partial M \times[0, d]$.

Theorem 1. If $\varepsilon$ and $m$ are positive numbers, then there are positive numbers $A$ and $T$ such that the following holds. If $M$ is a compact hyperbolic 3-manifold with totally geodesic boundary $Y$ with $\chi(Y) \geq-m$ and $\operatorname{injrad}(Y) \geq \varepsilon$, and $M$ contains a collar of depth $d \geq T$ about $\Sigma$, then the skinning map $\sigma_{M}$ has diameter less than $A e^{-d}$.

We pause to sketch the proof.

Consider the hyperbolic manifolds $\mathrm{qf}(X, \bar{Y})$ and $\mathrm{qf}(Y, \bar{Y})$. Very far out toward their $\bar{Y}$-ends, these manifolds are very nearly isometric. In fact, the proximity of the metrics decays exponentially in the distance from the convex core. Using foliations constructed by C. Epstein, the metrics near the $\bar{Y}$-ends of $\operatorname{qf}(X, \bar{Y})$ and $\operatorname{qf}(Y, \bar{Y})$ may be written down explicitly in terms of the Schwarzian derivatives associated to the projective structures on $\bar{Y}$, see Section 2. This allows us to explicitly glue the " $X$-side" of $\mathrm{qf}(X, \bar{Y})$ to the " $\bar{Y}$-side" of $\mathrm{qf}(Y, \bar{Y})$ to obtain a metric $\eta$ on $S \times \mathbb{R}$ which is hyperbolic away from a shallow gluing region of the form $S \times[n, n+1]$, see Figure 1. Calculations (in Sections 3.2.1 and 3.2.3) show that the resulting metric has sectional and traceless Ricci curvatures exponentially close to -1 and 0 , respectively. Moreover, the $L^{2}$-norm of the traceless Ricci curvature of this metric is exponentially small (see Section 3.2.2).

Given a hyperbolic manifold $M^{Y}$ with totally geodesic boundary $Y$ possessing a large collar about its boundary, we may glue the "X-side" of $\mathrm{qf}(X, \bar{Y})$ to a compact piece of $M^{Y}$ in the same way to obtain a metric $\omega$ on $M^{\circ}$ with the same curvature bounds, see Figure 2.

It is a theorem of Tian that a Riemannian metric on a closed 3-manifold whose sectional curvatures are very close to -1 and whose traceless Ricci curvature has very small $L^{2}$-norm is $\mathscr{C}^{2}$-close to a hyperbolic metric, where the proximity depends only on the curvatures and their norms and not the topology of the 3-manifold. As our manifold is noncompact, Tian's theorem is not directly applicable. A theorem of Brooks [7] allows us to circumvent this problem by performing a small quasiconformal deformation of $\mathrm{qf}(X, \bar{Y})$ to obtain a manifold covering a closed one, and we find that $\omega$ is $\mathscr{C}^{2}$-close to the convex cocompact manifold $M^{X} \cong M^{\circ}$ with conformal boundary $X$. See Section 5.

Now, the copy of $S \times\{n+1\}$ in $\left(M^{\circ}, \omega\right)$ is conformally equivalent to $\bar{Y}$, and, for large $n$, the proximity of the metrics implies that the corresponding surface in $M^{X}$ is 


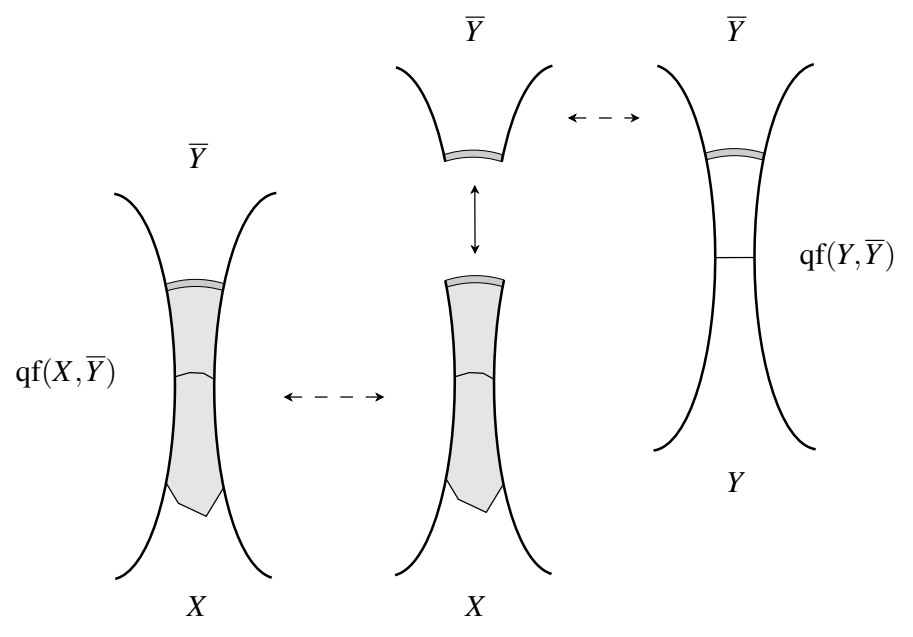

Figure 1: Building the metric $\eta$ on $S \times \mathbb{R}$.

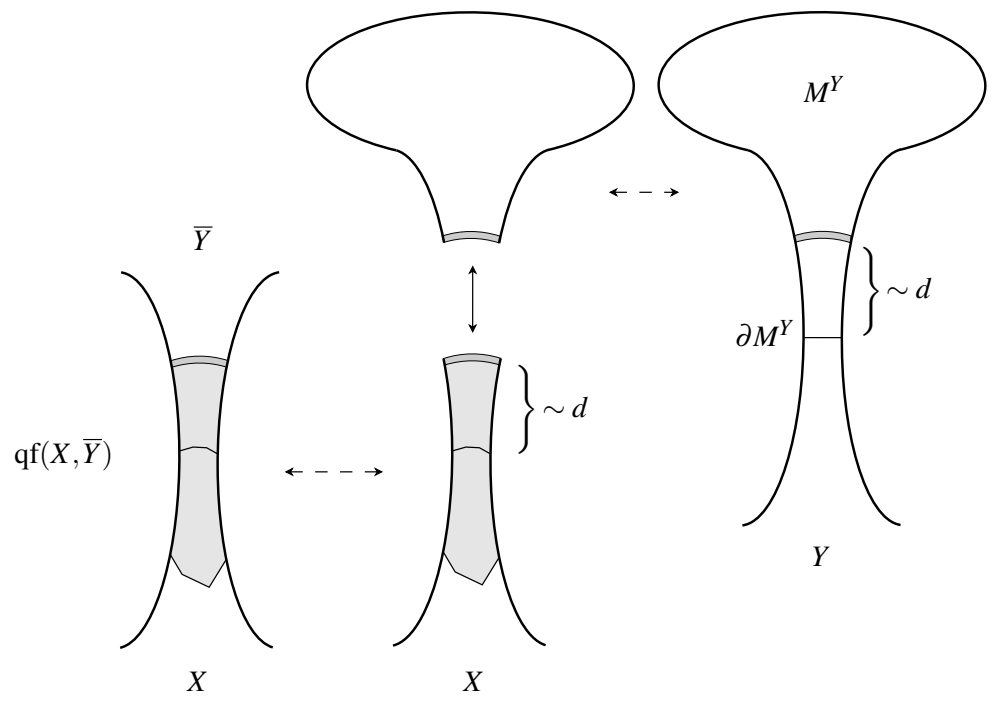

Figure 2: Building the metric $\eta$ on $M^{\circ}$. 
very close to both $\sigma_{M}(X)$ and $\bar{Y}$ in Teichmüller space. We conclude that the image of $\sigma_{M}$ lies in a small neighborhood of $\bar{Y}$.

Generalizations. The hypothesis bounding the injectivity radius is needed in the proof in two places: to bound the Sobolev norms of the Schwarzian derivatives of developing maps of the quasifuchsian manifolds, which is needed in the estimation of the curvatures of our model metric; and to guarantee that our model metric is Einstein on the 1-thin part, which is required by Tian's theorem. We suspect that one may dispense with this hypothesis, though our proof can not.

The dependence of the constants $A$ and $T$ on $\chi(\partial M)$ is likely necessary, though we do not have a sequence of examples to demonstrate this. In [12], the first author shows that there are manifolds with arbitrarily large diameter skinning maps, but the maximum depth of the collars about their geodesic boundaries tends to zero.

Skinning maps are defined for any orientable hyperbolic manifold with incompressible boundary, and we in fact establish the following generalization of Theorem 1.

If $Z$ is a Riemann surface, let $\operatorname{inj} \operatorname{rad}(Z)$ denote the injectivity radius of the hyperbolic metric in its conformal class.

Theorem 2. Let $M=M^{W}$ be a convex cocompact hyperbolic 3-manifold with freely indecomposable nonelementary fundamental group and conformal boundary $W$. Let $p: \operatorname{qf}(W, \bar{Y}) \rightarrow M$ be the covering map corresponding to $W$. There are constants $A, T$, and $K$ depending only on $\chi(W)$ and $\operatorname{inj} \operatorname{rad}(\bar{Y})$ such that the following holds.

If $d>T$ and $p$ embeds the $d$-neighborhood of the convex core of $\mathrm{qf}(W, \bar{Y})$ isometrically into $M$, then $\sigma_{M}$ has diameter no more than $A e^{-d}$ and the $K d$-neighborhood of the convex core of $\mathrm{qf}\left(V, \sigma_{M}(V)\right)$ isometrically embeds into $M^{V}$ for any $V$.

In particular, the manifold $M$ is acylindrical and the totally geodesic boundary has a large collar (of depth $\mathrm{Kd}$ ) in the corresponding hyperbolic structure on $\mathrm{M}$.

Note that $M$ being acylindrical would follow from any bound on the diameter of the skinning map, as cylindrical manifolds always have unbounded skinning maps.

The proof of Theorem 2 roughly follows the sketch given above. The construction of the Riemannian metric $\eta$ in the general setting is recorded below in Theorem 4, and may be of independent interest.

Miscellaneous notation. If $f$ and $F$ are functions of $t$, we use the Landau notation $f=\mathscr{O}(F)$ to mean that there is a constant $L$ such that $|f(t)| \leq L F(t)$ for all $t$. If $a$, $b, c, \ldots$ are objects, we write $f(t)=\mathscr{O}_{a, b, c, \ldots}(F(t))$ if $|f(t)| \leq L F(t)$ for a constant $L$ depending only on $a, b, c, \ldots$ We use the standard notation $W^{k, p}(\mathscr{X})$ for Sobolev spaces and follow the Einstein summation convention.

Acknowledgments. The authors thank Ken Bromberg, David Dumas, Hossein Namazi, Sean Paul, and Jeff Viaclovsky for helpful conversations. They also thank the referee for such careful readings and numerous useful suggestions. In particular, we are very grateful for the referee's many careful observations that improved our estimate from $A e^{-d / 2}$ to $A e^{-d}$. 


\section{Tian's theorem}

Theorem 3 (Tian [25]). There are numbers $C_{\text {Tian }} \geq 1$ and $\varepsilon_{\text {Tian }}>0$ such that the following holds. If $\varepsilon<\varepsilon_{\text {Tian }}$ and $(M, \omega)$ is a closed Riemannian 3-manifold with sectional curvatures pinched between $-1-\varepsilon$ and $-1+\varepsilon$, traceless Ricci curvature $\mathrm{Ric}^{\omega}+2 \omega=0$ on the 1-thin part, and

$$
\sqrt{\int_{M}\left\|\mathrm{Ric}^{\omega}+2 \omega\right\|_{\omega}^{2} \mathrm{~d} V_{\omega}} \leq \varepsilon
$$

then $M$ has a hyperbolic metric $\zeta$ such that $\|\omega-\zeta\|_{\mathscr{C}^{2}(M, \omega)} \leq C_{\text {Tian }} \varepsilon$, where $\|\cdot\|_{\mathscr{C}^{2}(M, \omega)}$ is the $\mathscr{C}^{2}-$ norm with respect to $\omega$.

The background metric $\omega$ defines the pointwise norm of any tensor on $M$, and the pointwise $\mathscr{C}^{2}$-norm of a smoothly varying bilinear form $b$ is defined by taking the supremum of the norm of $b$ and its first two covariant derivatives with respect to $\omega$. The norm $\|b\|_{\mathscr{C}^{2}(M, \omega)}$ is then the supremum of the pointwise norms.

\section{Hyperbolic metrics and Epstein surfaces}

Most of this section is a review of Section 6.1 of [6] and Sections 3.2-3.4 of [2].

Let $\Delta$ be the open unit disk in $\mathbb{C}$ parameterized by the variable $z=x+i y$. We model hyperbolic space $\mathbb{H}^{3}$ as $\Delta \times \mathbb{R}$ with the metric $g$ given by

$$
\mathrm{d} s^{2}=\frac{4 \cosh ^{2} t}{\left(1-|z|^{2}\right)^{2}} \mathrm{~d} x^{2}+\frac{4 \cosh ^{2} t}{\left(1-|z|^{2}\right)^{2}} \mathrm{~d} y^{2}+\mathrm{d} t^{2} .
$$

Note that $\Delta \times\{0\}$ is a totally geodesic hyperbolic plane. We encode $g$ in the matrix

$$
g=\left(g_{i j}\right)=\left(\begin{array}{ccc}
\lambda^{2} \cosh ^{2} t & 0 & 0 \\
0 & \lambda^{2} \cosh ^{2} t & 0 \\
0 & 0 & 1
\end{array}\right)
$$

where $\lambda=2 /\left(1-|z|^{2}\right)$.

Let $\Gamma^{Y}$ be a fuchsian group uniformizing $\bar{Y}$ via $\Delta / \Gamma^{Y}=\bar{Y}$. This gives us the fuchsian hyperbolic 3-manifold $\operatorname{qf}(Y, \bar{Y})=\mathbb{H}^{3} / \Gamma^{Y}$, and a we have a local expression for the hyperbolic metric on $\operatorname{qf}(Y, \bar{Y})$ in (2.1). We let $\mathscr{D}^{Y}$ be a compact fundamental domain for $\Gamma^{Y}$ acting on $\Delta$ whose interior contains zero.

We also want to consider the Poincaré ball model $\mathbb{B}^{3}$ of $\mathbb{H}^{3}$, with boundary the Riemann sphere $\widehat{\mathbb{C}}$. There is a unique isometry $\imath: \Delta \times \mathbb{R} \rightarrow \mathbb{B}^{3}$ which extends continuously to $\Delta \times\{ \pm \infty\}$, taking $\Delta \times\{\infty\}$ to $\Delta \subset \widehat{\mathbb{C}}$ by the identity map. Note that on $\Delta \times\{\infty\}$ this extension carries $\frac{\partial}{\partial t}$ to a vector pointing downward, or out of hyperbolic space.

Let $\varphi: \Delta \rightarrow \widehat{\mathbb{C}}$ be a univalent function, ${ }^{* *}$ let

$$
\mathscr{S} \varphi(z)=\left(\frac{\varphi_{z z}}{\varphi_{z}}\right)_{z}-\frac{1}{2}\left(\frac{\varphi_{z z}}{\varphi_{z}}\right)^{2}
$$

\footnotetext{
${ }^{* *} \mathrm{~A}$ function $\Delta \rightarrow \widehat{\mathbb{C}}$ is univalent if it is injective and holomorphic.
} 
be its Schwarzian derivative, and let $\|\mathscr{S} \varphi(z)\|=\left|\lambda^{-2} \mathscr{S} \varphi(z)\right|$. Let $M_{\varphi(z)}: \widehat{\mathbb{C}} \rightarrow \widehat{\mathbb{C}}$ be the osculating Möbius transformation to $\varphi$ at $z$ (the Möbius transformation with the same 2-jet as $\varphi$ at $z$ ). This uniquely extends to an isometry $M_{\varphi(z)}: \mathbb{B}^{3} \rightarrow \mathbb{B}^{3}$. There is then a map $\Phi: \Delta \times \mathbb{R} \rightarrow \mathbb{B}^{3}$ given by

$$
\Phi(z, t)=M_{\varphi(z)}(l(z, t))
$$

which also admits a continuous extension to $\Delta \times(-\infty, \infty]$ with $\Phi(z, \infty)=\varphi(z)$. We henceforth identify $\Delta$ with $\Delta \times\{\infty\}$, and identify both $\Delta \times \mathbb{R}$ and $\mathbb{B}^{3}$ with $\mathbb{H}^{3}$.

There is an orthonormal basis $\mathbf{e}_{1}, \mathbf{e}_{2}, \frac{\partial}{\partial t}$ for the tangent space to $\mathbb{H}^{3}$ at $(z, t)$ and an orthonormal basis for the tangent space to $\mathbb{H}^{3}$ at $\Phi(z, t)$ such that the derivative of $\Phi$ at $(z, t)$ is given by

$$
\left.\mathrm{D} \Phi\right|_{(z, t)}=\left(\begin{array}{ccc}
1+\frac{\|\mathscr{S} \varphi(z)\|}{e^{t} \cosh t} & 0 & 0 \\
0 & 1-\frac{\|\mathscr{S} \varphi(z)\|}{e^{t} \cosh t} & 0 \\
0 & 0 & 1
\end{array}\right) .
$$

(In [6], the eigenvalues of the matrix $\mathrm{D} \Phi-I$ are off by a factor of 4.) If we normalize (by conjugation in $\mathrm{PSL}_{2} \mathbb{C}$ ) so that $z=0$ and so that the osculating Möbius transformation at zero is the identity, we have

$$
\begin{aligned}
2 \cosh t \cdot \mathbf{e}_{1} & =\cos \left(\theta_{0}\right) \frac{\partial}{\partial x}+\sin \left(\theta_{0}\right) \frac{\partial}{\partial y} \\
2 \cosh t \cdot \mathbf{e}_{2} & =-\sin \left(\theta_{0}\right) \frac{\partial}{\partial x}+\cos \left(\theta_{0}\right) \frac{\partial}{\partial y}
\end{aligned}
$$

where $\theta_{0}$ is the argument of $\mathscr{S} \varphi(0)$, see Section 3.3 of [2].

The inequality $\|\mathscr{S} \varphi(z)\| \leq 3 / 2$ holds for univalent $\varphi$ by a celebrated theorem of Kraus [13] and Nehari [19], and so $\Phi$ is an orientation-preserving immersion on $\{(z, t) \in \Delta \times \mathbb{R} \mid t>\log \sqrt{2}\}$, by $(2.2)$.

The principal curvatures of $\Phi(\Delta \times\{t\})$ at $(z, t)$ are given by

$$
\kappa_{ \pm}(z, t)=\frac{1-(1 \pm 2\|\mathscr{S} \varphi(z)\|) e^{-2 t}}{1+(1 \pm 2\|\mathscr{S} \varphi(z)\|) e^{-2 t}}
$$

when this is defined, see Proposition 6.3 of [6]. If $\mathscr{S} \varphi(z)=1$, then $\kappa_{+}(z, t)=\operatorname{coth} t$ by continuity of the principal curvatures. These curvatures are positive provided $t>\log 2$, thanks to the Kraus-Nehari theorem, and so $\Phi(\Delta \times\{t\})$ is locally convex for such $t .^{\circ}$

We now specialize to univalent $\varphi$ associated to ends of hyperbolic 3-manifolds.

Let $M$ be a complete hyperbolic 3-manifold with conformally compact incompressible end $E=E^{M}$ compactified by the Riemann surface $\bar{Y}$. Let $\Gamma=\Gamma^{M} \subset \operatorname{PSL}_{2}(\mathbb{C})$ be a Kleinian group uniformizing $M$.

Blurring the distinction between ends and their neighborhoods, the end $E$ is homeomorphic to $S \times(0, \infty)$, and we pick isomorphisms $\pi_{1}(E) \leftarrow \pi_{1}(S) \rightarrow \Gamma^{\bar{Y}}$ compatible

\footnotetext{
${ }^{\circ}$ The statement in [6] that the $\Phi(\Delta \times\{t\})$ are convex when $t>0$ is an error.
} 
with the chosen marking of $\bar{Y}$. Choose a component $\mathscr{U}^{\bar{Y}}$ of the domain of discontinuity of $\Gamma^{M}$ corresponding to $E$, let $\varphi: \Delta \rightarrow \mathscr{U}^{\bar{Y}}$ be the $\pi_{1}(S)$-equivariant Riemann mapping, and let $\Phi: \Delta \times(\log 2, \infty] \rightarrow \mathbb{H}^{3} \cup \mathscr{U}^{\bar{Y}}$ be as above. By the above discussion, the map $\Phi$ is an immersion and the surfaces $\Phi(\Delta \times\{t\})$ are locally strictly convex.

Given a smooth surface $\mathscr{F}$ and a locally strictly convex immersion $f: \mathscr{F} \rightarrow \mathbb{H}^{3}$, there is an associated Gauss map $\mathfrak{g}: \mathscr{F} \rightarrow \widehat{\mathbb{C}}$, defined as follows. For any $w$ in $\mathscr{F}$, there is a neighborhood $U$ of $w$ on which $f$ is an embedding. Since $f$ is locally strictly convex, there is a unique geodesic ray emanating from $f(w)$ that is perpendicular to $f(U)$ and moves away from the center of curvature. This geodesic ray has a unique endpoint $g(w)$ in $\widehat{\mathbb{C}}$, and this defines a map $\mathfrak{g}: \mathscr{F} \rightarrow \widehat{\mathbb{C}}$.

Note that it follows from (2.2) that the Gauss map $\mathfrak{g}_{t}: \Delta \times\{t\} \rightarrow \widehat{\mathbb{C}}$ associated to the immersion $\Phi_{t}=\left.\Phi\right|_{\Delta \times\{t\}}$ is equal to the embedding $\varphi \circ p_{t}$, where $p_{t}: \Delta \times\{t\} \rightarrow \Delta$ is the projection $p_{t}(z, t)=z$. In particular, $\mathfrak{g}_{t}$ descends to an injective map on $\Delta / \pi_{1}(S)$.

Let $\Xi: \Delta / \pi_{1}(S) \times(\log 2, \infty] \rightarrow E \cup \bar{Y}$ be the immersion induced by $\Phi$ and let $\Xi_{t}$ be the restriction of $\Xi$ to $\Delta / \pi_{1}(S) \times\{t\}$.

We claim that $\Xi$ is a diffeomorphism. ${ }^{\circ 0}$

To see this, first note that since $\Xi_{\infty}$ is a diffeomorphism and $S$ is compact, the implicit function theorem provides a $t_{0}$ such that $\Xi$ is an embedding when restricted to $\Delta / \pi_{1}(S) \times\left[t_{0}, \infty\right]$.

Suppose that $\Xi$ is not a diffeomorphism, and let $t_{1}$ be the largest $t$ in $(\log 2, \infty)$ such that $\Xi$ is not injective on $\Delta / \pi_{1}(S) \times[t, \infty)$. So $\Xi_{t_{1}}$ is not injective, and we have points $a$ and $b$ in $\Delta / \pi_{1}(S) \times\left\{t_{1}\right\}$ for which $\Xi_{t_{1}}(a)=\Xi_{t_{1}}(b)$. Local strict convexity implies that $\Xi_{t_{1}}$ must have a self-tangency at these points, or else there would be a slightly later time $t_{2}$ at which $\Xi_{t_{2}}$ failed to be an embedding. Furthermore, the normal vectors pointing away from the centers of curvature must agree, or else there would again be a slightly later time when $\Xi_{t}$ failed to embed. Lifting the map $\Xi_{t_{1}}$ to the map $\Phi_{t_{1}}$, we find distinct points $\widetilde{a}$ and $\widetilde{b}$ in $\Delta \times\left\{t_{1}\right\}$ such that $\mathfrak{g}_{t_{1}}(\widetilde{a})=\mathfrak{g}_{t_{1}}(\widetilde{b})$, contradicting injectivity of the Gauss map $\mathfrak{g}_{t_{1}}$. We conclude that $\Xi$ is a diffeomorphism.

Let $\mathscr{E}_{t}=\mathscr{E}_{t} M$ be the image of $\Xi_{t}$. We call the $\mathscr{E}_{t}$ Epstein surfaces, in honor of their study by C. Epstein [9], who calls them Weingarten surfaces.

\section{Gluing hyperbolic metrics}

We prove the following gluing theorem for hyperbolic manifolds, which says roughly the following. If two hyperbolic manifolds $M^{1}$ and $M^{2}$ contain separating product regions $S \times I$ isometric to products taken from far out in conformally compact $\bar{Y}$-ends of two other hyperbolic manifolds $N^{1}$ and $N^{2}$, then these regions cut $M^{1}$ and $M^{2}$ into convex pieces $\mathscr{A}^{1}$ and $\mathscr{A}^{2}$ and concave pieces $\mathscr{B}^{1}$ and $\mathscr{B}^{2}$. The theorem says that one may glue $\mathscr{A}^{1}$ to $\mathscr{B}^{2}$ so that the resulting manifold admits a Riemannian metric that is very nicely behaved near the gluing site and hyperbolic elsewhere. In particular, if the resulting manifold is closed, this Riemannian metric satisfies the hypotheses of Tian's theorem, and is thus close to the unique hyperbolic metric. See Figure 3.

\footnotetext{
${ }^{\circ}$ The argument given here is implicit in Sections 3 and 6 of [6].
} 


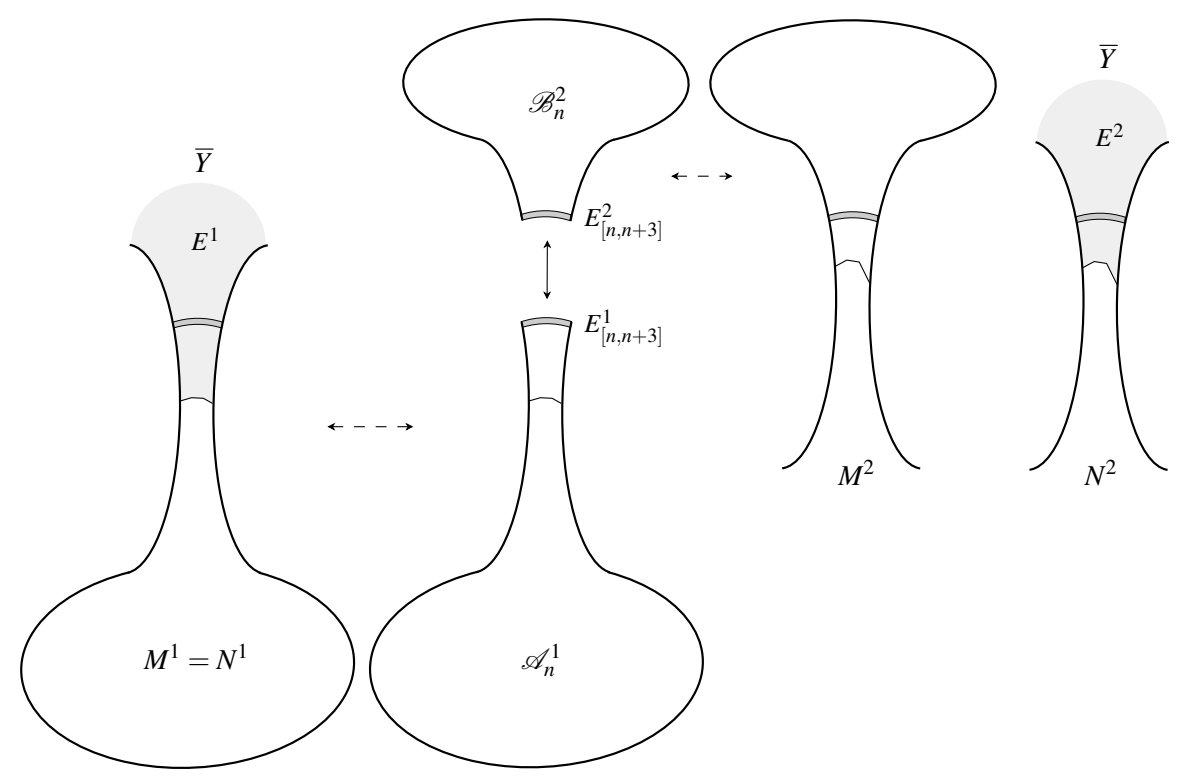

Figure 3: Building $\mathfrak{M}$ with the metric $\eta$. The case $M^{1}=N^{1}$ is shown for simplicity.

Theorem 4 (Model manifold for convex cocompact gluings). Given positive numbers $\varepsilon$ and $m$, there is a constant $A_{0}=A_{0}(m, \varepsilon)$ such that the following holds.

Let $\bar{Y}$ be a marked Riemann surface with $\chi(\bar{Y}) \geq-m$ and $\operatorname{injrad}(\bar{Y}) \geq \varepsilon$.

For i in $\{1,2\}$, let $\left(N^{i}, \zeta^{i}\right)$ be a complete hyperbolic 3-manifold with a conformally compact (marked) incompressible end $E^{i}$ conformally compactified by $\bar{Y}$. Let $\mathscr{E}_{t}^{i}$ be the foliation of $E^{i}$ by Epstein surfaces. For $\log 2<a<b$, let $E_{[a, b]}^{i}$ be the compact region of $E^{i}$ bounded by $\mathscr{E}_{a}^{i}$ and $\mathscr{E}_{b}$.

Let $\left(M^{i}, \xi^{i}\right)$ be a complete hyperbolic manifold containing a separating submanifold isometric to $E_{[n, n+3]}^{i}$. For $t$ in $[a, b]$, let $\mathscr{A}_{t}^{i}$ be the closure of the convex component of $M^{i}-\mathscr{E}_{t}^{i}$, and let $\mathscr{B}_{t}^{i}$ be the closure of the concave component.

Let $\mathfrak{M}$ be the topological manifold obtained by gluing $\mathscr{A}_{n}^{1}$ to $\mathscr{B}_{n}^{2}$ along their boundaries respecting the marking of $\bar{Y}$.

Then there is a Riemannian metric $\eta$ on $\mathfrak{M}$ that satisfies the following.

1. The inclusions of $\left(\mathscr{A}_{n}^{1}, \xi^{1}\right)$ and $\left(\mathscr{B}_{n+3}^{2}, \xi^{2}\right)$ into $(\mathfrak{M}, \eta)$ are isometric embeddings.

2. The sectional curvatures of $\eta$ are within $A_{0} e^{-2 n}$ of -1 .

3. The $L^{2}$-norm of the traceless Ricci curvature of $\eta$ is no more than $A_{0} e^{-n}$.

4. The injectivity radius of $\eta$ on $E_{[n, n+3]}^{2}$ is at least $\left(1-A_{0} e^{-2 n}\right) \operatorname{inj} \operatorname{rad}(\bar{Y}) \cosh n$.

Proof. We begin by assuming that $M^{1}=N^{1}$ and that $M^{2}=N^{2}=\mathrm{qf}(Y, \bar{Y})$. 


\subsection{The proof of Theorem 4 when $M^{1}=N^{1}$ and that $M^{2}=N^{2}=$ $\operatorname{qf}(Y, \bar{Y})$.}

Let $s_{0}(t)$ be a smooth nonincreasing function on $\mathbb{R}$ such that $s_{0}(t)=1$ when $t \leq 0$ and $s_{0}(t)=0$ when $t \geq 1$. Let $s_{n}(t)=s_{0}(t-n)$. As the Sobolev norms of the $s_{n}$ are independent of $n$, we write $s(t)$ for $s_{n}(t)$ and let context dictate $n$.

Let $\Phi: \Delta \times \mathbb{R} \rightarrow \mathbb{B}^{3}$ be the map associated to $E^{1}$ constructed in Section 2, and let $h=\Phi^{*} g$ be the pullback of the hyperbolic metric via $\Phi$. We interpolate between the hyperbolic metrics $g$ and $h$ on $\Delta \times[n, n+1]$ using the metric

$$
\eta=(1-s(t)) g+s(t) h
$$

which descends to a metric on $S \times[n, n+1]$ that we also call $\eta$, see Figure 1 .

By (2.2), (3.1), and the Kraus-Nehari theorem, we have the following proposition, which provides Part 4 of Theorem 4.

Proposition 5. The identity map $\imath:(S \times[n, n+1], g) \rightarrow(S \times[n, n+1], \eta)$ is $\left(1+\mathscr{O}_{S}\left(e^{-2 n}\right)\right)$-bilipschitz with Jacobian determinant $1+\mathscr{O}_{S}\left(e^{-4 n}\right)$.

To prove the other parts of Theorem 4 when $M^{1}=N^{1}$ and $M^{2}=N^{2}=\operatorname{qf}(Y, \bar{Y})$, we begin by showing that the traceless Ricci curvature of our metric $\eta$ is on the order of $e^{-2 n}$. The region where $\eta$ is nonhyperbolic has volume on the order of $e^{2 n}$, and so it will follow that the $L^{2}$-norm of the traceless Ricci curvature is on the order of $e^{-n}$.

Since we are in dimension three, having Ricci curvature on the order of $e^{-2 n}$ implies that the difference between the sectional curvatures and -1 is on the order of $e^{-2 n}$ as well.

The intuition for the estimate of the Ricci curvature is as follows. The Ricci curvature measures the infinitesimal defect in volume of a sharp geodesic cone compared to the corresponding Euclidean cone: the volume element of a metric $\omega$ at a point $p$ admits an asymptotic expansion in $\omega$-geodesic normal coordinates

$$
\mathrm{d} V_{\omega}=\left(1-\frac{1}{6} \operatorname{Ric}^{\omega}(\mathbf{u}) \varepsilon^{2}+\mathscr{O}\left(\varepsilon^{3}\right)\right) \varepsilon^{2} \mathrm{~d} \varepsilon \mathrm{d} A(\mathbf{u})
$$

where $\operatorname{Ric}^{\omega}$ is the Ricci curvature of $\omega$ considered a quadratic form, and $\mathrm{d} A(\mathbf{u})$ is the canonical spherical measure on the unit tangent space $\mathrm{T}_{p}^{1} M$, see section 3.H.4 of [10]. Our metric $\eta$ is obtained by gluing two hyperbolic metrics on $S \times \mathbb{R}$ together fiberwise. The original metrics on the fibers are exponentially close, as are the original normal curvatures to the fibers, and so, after interpolating to obtain $\eta$, the volumes of cones are disturbed an exponentially small amount. One may try to make this precise using (3.2) and Proposition 5. This shows that the Ricci curvatures are close, but depends on the precise rate of decay of the $\mathscr{O}\left(\varepsilon^{3}\right)$ term in $\mathrm{d} V_{\eta}$. Fortunately, the Ricci curvatures are fairly easy to estimate directly.

\subsection{Bounds on curvatures}

If $\omega=\omega_{i j} \mathrm{~d} x^{i} \mathrm{~d} x^{j}$ is a Riemannian metric, we have Christoffel symbols

$$
\Gamma_{i j}^{\ell}(\omega)=\frac{1}{2} \omega^{k \ell}\left(\frac{\partial}{\partial x^{i}} \omega_{k j}+\frac{\partial}{\partial x^{j}} \omega_{i k}-\frac{\partial}{\partial x^{k}} \omega_{i j}\right),
$$


where $\left(\omega^{i j}\right)=\left(\omega_{i j}\right)^{-1}$.

\subsubsection{Bounding the Ricci curvature}

The Ricci curvature tensor $\operatorname{Ric}^{\omega}=R_{i j}^{\omega} \mathrm{d} x^{i} \mathrm{~d} x^{j}$ of a metric $\omega$ in coordinates $x^{i}$ is given by

$$
R_{i j}^{\omega}=\left(\frac{\partial \Gamma_{i j}^{\ell}}{\partial x^{\ell}}-\frac{\partial \Gamma_{i \ell}^{\ell}}{\partial x^{j}}+\Gamma_{i j}^{\ell} \Gamma_{\ell m}^{m}-\Gamma_{i \ell}^{m} \Gamma_{j m}^{\ell}\right)(\omega) .
$$

Theorem 6. If $\mathbf{u}$ is an $\eta$-unit vector at $(z, t)$ in $S \times[n, n+1]$, then

$$
\operatorname{Ric}^{\eta}(\mathbf{u})-\operatorname{Ric}^{g}(\mathbf{u})=\mathscr{O}\left(e^{-2 n}\right) .
$$

Theorem 6 follows immediately from the following theorem.

Theorem 7. We have $\|\eta-g\|_{\mathscr{C}^{2}(S \times[n, n+1], g)}=\mathscr{O}\left(e^{-2 n}\right)$.

Proof. Since the norms $\|\mathscr{S} \varphi(z)\|$ and arguments $\arg (\mathscr{S} \varphi(z))$ are smooth functions away from the zeroes of $\mathscr{S} \varphi$, and the set of points $(z, t)$ such that $\mathscr{S} \varphi(z)=0$ is a finite set of lines in $S \times \mathbb{R}$, we restrict attention to points $(z, t)$ such that $\mathscr{S} \varphi(z) \neq 0$.

Let $\mathscr{Q}(\bar{Y})$ be the vector space of holomorphic quadratic differentials on $\bar{Y}$. By the Kraus-Nehari theorem, the subset of $\mathscr{Q}(\bar{Y})$ consisting of Schwarzian derivatives of developing maps of Kleinian projective structures on $Y$ is compact, see [4]. So there is a number $\mathscr{B}_{0}=\mathscr{B}_{0}(Y)$ bounding the values and first few partial derivatives of the norms $\|\mathscr{S} \varphi(z)\|$ and arguments $\arg (\mathscr{S} \varphi(z))$ on the fundamental domain $\mathscr{D}^{Y}$. In other words, the functions $\|\mathscr{S} \varphi(z)\|$ and $\arg (\mathscr{S} \varphi(z))$ have Sobolev norms $\|\cdot\|_{W^{2, \infty}\left(\mathscr{D}^{Y}\right)}$ at most $\mathscr{B}_{0}$. In fact, if we fix a compact subset $\mathscr{X}$ of $\mathscr{T}(\bar{Y})$ containing $\bar{Y}$, we obtain a uniform bound $\mathscr{B}_{1}=\mathscr{B}_{1}(\mathscr{X})$ on these Sobolev norms over all of $\mathscr{X}$. As the thick part of the moduli space $\mathscr{M}(\bar{Y})$ is compact [18], the action of the mapping class group $\operatorname{Mod}(S)$ on $\mathscr{T}(\bar{Y})$ provides a bound $\mathscr{B}=\mathscr{B}(\chi(S), \operatorname{injrad}(Y))$ on these Sobolev norms over the entire thick part of $\mathscr{T}(\bar{Y})$.

The notation $\mathscr{O}(\cdot)$ will now always mean $\mathscr{O}_{\chi(S), \operatorname{injrad}(Y)}(\cdot)$.

Consider a point $(w, t)$ in $\Delta \times[0, \infty)$ with $\mathscr{S} \varphi(w) \neq 0$. Composing $\varphi$ on both sides with Möbius transformations, we may assume that $w=0$ and $M_{\varphi(0)}=$ Id.

We work with a small ball $B \subset \mathscr{D}^{Y} \subset \Delta$ centered at 0 containing no zeroes of $\mathscr{S} \varphi$.

Let $z$ be a point of $B$, and let $\psi_{z}$ be the hyperbolic element of PSL ${ }_{2} \mathbb{C}$ stabilizing $\Delta$ that carries 0 to $z$ and whose axis in $\Delta$ contains 0 . By the invariance of the Schwarzian, we have

$$
\mathscr{S}\left(\varphi \circ \psi_{z}\right)(0)=\mathscr{S} \varphi\left(\psi_{z}(0)\right) \psi_{z}^{\prime}(0)^{2}=\mathscr{S} \varphi(z) \psi_{z}^{\prime}(0)^{2} .
$$

Note that $\psi_{z}^{\prime}(0)=1 /\left(1-|z|^{2}\right)$. We may postcompose $\varphi \circ \psi_{z}$ with a Möbius transformation to ensure that the osculating Möbius transformation to $\varphi \circ \psi_{z}$ at 0 is the identity, and this has no effect on the Schwarzian. Let

$$
\theta_{z}=\arg (\mathscr{S} \varphi(z))-\arg \left(\psi_{z}^{\prime}(0)^{2}\right) .
$$


A change of variables allows us to assume that $\theta_{0}=0$. Let

$$
A_{z}=\left(\begin{array}{ccc}
\cos \left(\theta_{z}\right) & -\sin \left(\theta_{z}\right) & 0 \\
\sin \left(\theta_{z}\right) & \cos \left(\theta_{z}\right) & 0 \\
0 & 0 & 1
\end{array}\right)
$$

Consider coordinates $u^{1}=\cosh (t) x, u^{2}=\cosh (t) y$, and $u^{3}=t$. In these coordinates, the metric $g$ is given by

$$
g=\left(g_{i j}\right)=\left(\begin{array}{ccc}
\lambda^{2} & 0 & 0 \\
0 & \lambda^{2} & 0 \\
0 & 0 & 1
\end{array}\right)
$$

where $\lambda=2 /\left(1-|z|^{2}\right)$.

By (2.3), we have

$$
\left.\mathrm{D} \Phi\right|_{(z, t)}=A_{z}\left(\begin{array}{ccc}
1+\frac{\|\mathscr{S} \varphi(z)\|}{e^{t} \cosh t} & 0 & 0 \\
0 & 1-\frac{\|\mathscr{S} \varphi(z)\|}{e^{t} \cosh t} & 0 \\
0 & 0 & 1
\end{array}\right) A_{z}^{-1}
$$

in $B \times \mathbb{R}$ with respect to the orthonormal basis $\frac{1}{\lambda} \frac{\partial}{\partial u^{1}}, \frac{1}{\lambda} \frac{\partial}{\partial u^{2}}, \frac{\partial}{\partial u^{3}}$ to $\mathbb{H}^{3}$ at $(z, t)$ and an orthonormal basis at $\Phi(z, t)$. Note that $\left(g_{i j}\right)$, a scalar matrix when restricted to the subspace $\frac{\partial}{\partial u^{1}}$ and $\frac{\partial}{\partial u^{2}}$, commutes with the matrix $\mathrm{D} \Phi^{T}=\mathrm{D} \Phi$.

Writing the metric $\eta=(1-s(t)) g+s(t) h$ in these coordinates, we have

$$
\begin{aligned}
\left(\eta_{i j}\right) & =(1-s(t))\left(g_{i j}\right)+s(t) \mathrm{D} \Phi^{T} \cdot\left(g_{i j}\right) \cdot \mathrm{D} \Phi \\
& =(1-s(t))\left(g_{i j}\right)+s(t) \cdot\left(g_{i j}\right) \cdot(\mathrm{D} \Phi)^{2} .
\end{aligned}
$$

Expanding this, we have

$$
\left(\eta_{i j}\right)=\left(g_{i j}\right)+\frac{1}{e^{t} \cosh t} \cdot s(t) \lambda^{2}\|\mathscr{S} \varphi(z)\| \cdot P,
$$

where

$$
P=\left(\begin{array}{ccc}
2 \cos \left(2 \theta_{z}\right)+\frac{\|\mathscr{S} \varphi(z)\|}{e^{t} \cosh t} & 2 \sin \left(2 \theta_{z}\right) & 0 \\
2 \sin \left(2 \theta_{z}\right) & -2 \cos \left(2 \theta_{z}\right)+\frac{\|\mathscr{S} \varphi(z)\|}{e^{t} \cosh t} & 0 \\
0 & 0 & 0
\end{array}\right) .
$$

Proposition 8. For all $i, j, k$, and $\ell$, we have

$$
\left|\eta_{i j}-g_{i j}\right|=\mathscr{O}\left(e^{-2 n}\right)
$$


$\left|\frac{\partial}{\partial x^{k}} \eta_{i j}-\frac{\partial}{\partial x^{k}} g_{i j}\right|=\mathscr{O}\left(e^{-2 n}\right)$,

and

$$
\left|\frac{\partial}{\partial x^{\ell}} \frac{\partial}{\partial x^{k}} \eta_{i j}-\frac{\partial}{\partial x^{\ell}} \frac{\partial}{\partial x^{k}} g_{i j}\right|=\mathscr{O}\left(e^{-2 n}\right),
$$

in coordinates $u^{1}=\cosh (t) x, u^{2}=\cosh (t) y$, and $u^{3}=t$ on $S \times[n, n+1]$.

Proof. The estimates hold on $B$ by inspection of (3.10) and (3.11), and, since the zeroes of $\mathscr{S} \varphi$ are isolated and our metrics are smooth, they hold on all of $S \times[n, n+1]$ by continuity.

Now, in the coordinates $u^{i}$, the first few derivatives of the $g_{i j}$ and $g^{i j}$ are $\mathscr{O}(1)$, and so Proposition 8 implies that $\|\eta-g\|_{\mathscr{C}^{2}(S \times[n, n+1], g)}=\mathscr{O}\left(e^{-2 n}\right)$, by definition of the $\mathscr{C}^{2}$-norm.

This completes the proof of Theorem 7 (and hence of Theorem 6).

\subsection{2 $\quad L^{2}$-norm of the traceless Ricci curvature}

To estimate the $L^{2}$-norm of the traceless Ricci curvature of $\eta$, we begin by estimating the volume of the nonhyperbolic part.

Lemma 9. We have

$$
\int_{S \times[n, n+1]} 1 \mathrm{~d} V_{\eta} \leq-18 \pi \chi(S) e^{2 n}
$$

Proof. Let $l:(S \times[n, n+1], g) \rightarrow(S \times[n, n+1], \eta)$ be the identity map. By (2.2), the Jacobian determinant of $t$ at $(z, t)$ is

$$
|\operatorname{Jac} \imath|=1-\left(\frac{\|\mathscr{S} \varphi(z)\|}{e^{t} \cosh t}\right)^{2} .
$$

So

$$
\begin{aligned}
\int_{S \times[n, n+1]} 1 \mathrm{~d} V_{\eta} & =\int_{S \times[n, n+1]}|\operatorname{Jac} \imath| \mathrm{d} V_{g} \\
& \leq \int_{S \times[n, n+1]} 1 \mathrm{~d} V_{g} \\
& =\int_{\mathscr{D}^{Y} \times[n, n+1]} \sqrt{\operatorname{det} g} \mathrm{~d} x \mathrm{~d} y \mathrm{~d} t \\
& =\int_{\mathscr{D}^{Y} \times[n, n+1]} \lambda^{2} \cosh ^{2} t \mathrm{~d} x \mathrm{~d} y \mathrm{~d} t \\
& =\int_{S}\left(\int_{[n, n+1]} \cosh ^{2} t \mathrm{~d} t\right) \mathrm{d} A_{Y} \\
& \leq-2 \pi \chi(S) e^{2 n+2} \\
& \leq-18 \pi \chi(S) e^{2 n} .
\end{aligned}
$$


For an $\eta$-unit vector $\mathbf{u}$ at $(z, t)$, we have

$$
\operatorname{Ric}^{\eta}(\mathbf{u})+2 \eta(\mathbf{u})=\operatorname{Ric}^{g}(\mathbf{u})+2 g(\mathbf{u})+\mathscr{O}\left(e^{-2 t}\right)=\mathscr{O}\left(e^{-2 t}\right),
$$

by Theorem 6 . So there is a constant $A_{1}=A_{1}(\chi(S), \operatorname{injrad}(Y))$ such that

$$
\left\|\operatorname{Ric}^{\eta}+2 \eta\right\|_{\eta} \leq A_{1} e^{-2 n}
$$

Lemma 10. We have

$$
\sqrt{\int_{M}\left\|\operatorname{Ric}^{\eta}+2 \eta\right\|_{\eta}^{2} \mathrm{~d} V_{\eta}} \leq-18 \pi A_{1} \chi(S) e^{-n}
$$

Proof. Since $\eta$ is hyperbolic away from $S \times[n, n+1]$, Lemma 9 and (3.15) give us

$$
\begin{aligned}
\sqrt{\int_{M}\left\|\operatorname{Ric}^{\eta}+2 \eta\right\|_{\eta}^{2} \mathrm{~d} V_{\eta}} & \leq \sqrt{\int_{S \times[n, n+1]} A_{1}^{2} e^{-4 n} \mathrm{~d} V_{\eta}} \\
& =A_{1} e^{-2 n} \sqrt{\int_{S \times[n, n+1]} 1 \mathrm{~d} V_{\eta}} \\
& \leq A_{1} e^{-n} \sqrt{-18 \pi \chi(S)} \\
& \leq-18 \pi A_{1} \chi(S) e^{-n}
\end{aligned}
$$

\subsubsection{Sectional curvatures}

In dimension three, the sectional curvatures are determined by the Ricci curvatures. More specifically, if $\mathbf{u}, \mathbf{v}$, and $\mathbf{w}$ are orthonormal tangent vectors at a point in a 3manifold, we have

$$
2 K(\mathbf{u}, \mathbf{v})=\operatorname{Ric}(\mathbf{u})-\operatorname{Ric}(\mathbf{w})+\operatorname{Ric}(\mathbf{v}) .
$$

Theorem 6 and (3.17) give us

$$
\left.2 K^{\eta}(\mathbf{u}, \mathbf{v})\right|_{(z, t)}=-2+2-2+\mathscr{O}\left(e^{-2 t}\right)
$$

for any $\eta$-orthonormal vectors $\mathbf{u}$ and $\mathbf{v}$ at any $(z, t)$ in $\Delta \times[n, n+1]$. So

$$
\left.K^{\eta}(\mathbf{u}, \mathbf{v})\right|_{(z, t)}=-1+\mathscr{O}\left(e^{-2 n}\right)
$$

for all $(z, t)$, since $K^{\eta}=-1$ on $M-(\Delta \times[n, n+1])$. So there is a constant $A_{2}=$ $A_{2}(\chi(S), \operatorname{injrad}(Y)) \geq A_{1}$ such that

$$
-1-A_{2} e^{-2 n} \leq K^{\eta} \leq-1+A_{2} e^{-2 n}
$$

Let $A_{3}=\max \left\{-18 \pi A_{1} \chi(S), A_{2}, 1\right\}$.

Setting $A_{0}=A_{3}$ completes the proof of Theorem 4 in the case when $M^{1}=N^{1}$ and that $M^{2}=N^{2}=\mathrm{qf}(Y, \bar{Y})$. 


\subsection{The proof of Theorem 4 in the remaining cases}

Since the gluing takes place locally on the region $E_{[n, n+1]}^{2}$, the proof above provides all of the cases where $N^{2}=\mathrm{qf}(Y, \bar{Y})$. The cases when $N^{1}=\mathrm{qf}(Y, \bar{Y})$ are then obtained by replacing $s(t)$ with $1-s(t)$ in the proof. The general case is then obtained as follows. Let $E$ be the $\bar{Y}$-end of $\mathrm{qf}(Y, \bar{Y})$. First glue $\mathscr{A}_{n+1}^{1}$ to $E_{[n, \infty)} \subset \mathrm{qf}(Y, \bar{Y})$ along $E_{[n, n+1]}$ as above. Then glue $\mathrm{qf}(Y, \bar{Y})-E_{[n+3, \infty)}$ to $\mathscr{B}_{n+2}^{2}$ along $E_{[n+2, n+3]}$ as above. The resulting manifolds both contain an isometric copy of $E_{[n+1, n+2]}$, and a simple cut and paste completes the proof of the general case.

\section{Curvatures of surfaces and normal projections}

Letting $w=x_{1}+i x_{2}$, we compactify hyperbolic space by attaching the Riemann sphere $\widehat{\mathbb{C}}$ via the upper half-space model

$$
\mathbb{H}^{3}=\left\{\left(x_{1}, x_{2}, x_{3}\right) \in \mathbb{R}^{3} \mid x_{3}>0\right\} .
$$

Let $\mathscr{F}$ be a smooth surface in $\mathbb{H}^{3}$ equipped with a smooth unit normal field, and let $q$ be in $\mathscr{F}$. Applying an element of $\mathrm{PSL}_{2}(\mathbb{C})$, we assume that $q=(0,0,1)$, that the unit normal to $\mathscr{F}$ at $q$ is $-\mathbf{k}$, and that the principal directions at $q$ are $\mathbf{i}$ and $\mathbf{j}$. Let $v$ be the normal projection (or Gauss map) of $\mathscr{F}$ to $\widehat{\mathbb{C}}$ that sends each point of $\mathscr{F}$ to the point of $\widehat{\mathbb{C}}$ at the end of the geodesic ray given by our normal field. Picking an orthonormal basis for $\mathrm{T}_{q} \mathscr{F}$ along its principal directions and the usual basis for $\mathrm{T}_{0} \widehat{\mathbb{C}}$, the derivative of $v$ at $q$ is given by

$$
\mathrm{D} v_{q}=\left(\begin{array}{cc}
\frac{1+\kappa_{1}}{2} & 0 \\
0 & \frac{1+\kappa_{2}}{2}
\end{array}\right)
$$

where the $\kappa_{i}$ are the principal curvatures of $\mathscr{F}$ at $q$. Our convention is that normal curvatures are positive when the surface is curving away from the normal vector.

Lemma 11. Let $S \times[0, \infty)$ be a closed smoothly concave neighborhood of a convex cocompact end $E$ of a hyperbolic manifold and let $Z$ be the conformal boundary at $E$. If the principal curvatures of $\mathscr{G}=S \times\{0\}$ are within $\varepsilon$ of 1 for some $0<\varepsilon<1$, then $\mathscr{G}$ and $Z$ are $(1+\varepsilon)^{2}$-quasiconformal.

Proof. Lift $\mathscr{G}$ to a surface $\widetilde{\mathscr{G}}$ in $\mathbb{H}^{3}$ and normalize as above so that the derivative of the normal projection at a point $q$ in $\widetilde{\mathscr{G}}$ is

$$
\mathrm{D} v_{q}=\left(\begin{array}{cc}
\frac{1+\kappa_{1}(q)}{2} & 0 \\
0 & \frac{1+\kappa_{2}(q)}{2}
\end{array}\right)
$$

where the $\kappa_{i}(q)$ are the principal curvatures of $\widetilde{\mathscr{G}}$ at $q$. The usual Euclidean metrics on the tangent spaces $\mathrm{T}_{q} \widetilde{\mathscr{G}}$ and $\mathrm{T}_{0} \widehat{\mathbb{C}}$ are conformally compatible with the Riemannian metrics on $\widetilde{G}$ and $\widetilde{Z}$, respectively. Since the dilatation of the linear map D $v_{q}$ is at most $(1+\varepsilon / 2) /(1-\varepsilon / 2)<(1+\varepsilon)^{2}$ at the origin, the dilatation of the quasiconformal map $v$ is no more than $(1+\varepsilon)^{2}$ at $q$, see Chapter 1 of [1]. 
Lemma 12. Let $\mathrm{qf}(W, Z)$ be a quasifuchsian manifold, let $\mathscr{S} \varphi$ be the Schwarzian derivative of the developing map $\varphi: \Delta \rightarrow \mathscr{U}^{Z}$, and let $\mathscr{E}_{t}$ be the Epstein surface at time $t$ in the $Z$-end of $\mathrm{qf}(W, Z)$. If $t \geq \log 9$, then the principal curvatures $\kappa_{ \pm}(z, t)$ of $\mathscr{E}_{t}$ at $(z, t)$ satisfy

$$
\left|\kappa_{ \pm}(z, t)-1\right| \leq 9 e^{-2 t}
$$

Proof. This follows immediately from (2.4) and the Kraus-Nehari Theorem.

\section{Proof of Theorem 2}

Proof. Let $M^{W} \cong M$ be a convex cocompact hyperbolic manifold with conformal incompressible boundary $W$, let $p: \operatorname{qf}(W, \bar{Y}) \rightarrow M^{W}$ be the covering map corresponding to $W$, and assume that $p$ embeds the $d$-neighborhood of the convex core of $\operatorname{qf}(W, \bar{Y})$ isometrically into $M^{W}$. Let $n=\lfloor d\rfloor-8$. We make the crude choice of 8 to be sure that $S \times[n, n+3]$ avoids the thin part of $M^{W}$.

First assume that $X$ is such that the $2 n$-neighborhood of the convex core of $\mathrm{qf}(X, \bar{Y})$ together with the $\bar{Y}$-end of $\operatorname{qf}(X, \bar{Y})$ isometrically embeds into a convex cocompact hyperbolic 3-manifold $N^{1}$ with conformal boundary $\bar{Y}$, see Figure 4. Letting $M^{1}=N^{1}$, $N^{2}=\mathrm{qf}(W, \bar{Y})$, and $M^{2}=M$, let $\mathfrak{M}, \eta$, and $A_{0}=A_{0}(\chi(S), \operatorname{injrad}(Y))$ be as in Theorem 4. Note that while the topology of $\mathfrak{M}$ depends on $X$, our estimates do not. Since $\mathfrak{M}$ is closed, it follows from Tian's theorem that there is a $T=T(\chi(S), \operatorname{injrad}(Y)) \geq \log 9$ such that, when $n \geq T$, the metric $\eta$ on $\mathfrak{M}$ is within $C_{\text {Tian }} A_{0} e^{-n}<1 / 2$ of a hyperbolic

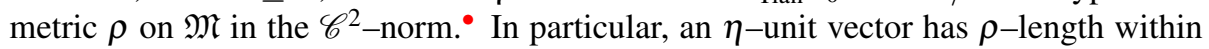
$C_{\text {Tian }} A_{0} e^{-n}$ of 1 . The metrics are then $\left(1+A_{4} e^{-n}\right)$-bilipschitz for $A_{4}=C_{\text {Tian }} A_{0}$. We summarize this discussion in a proposition.

Proposition 13. The metrics $\eta$ and $\rho$ satisfy

$$
\|\eta-\rho\|_{\mathscr{C}^{2}(\mathfrak{M}, \eta)} \leq A_{4} e^{-n}
$$

and are therefore $\left(1+A_{4} e^{-n}\right)$-bilipschitz.

Let $\mathfrak{M}_{\eta}=(\mathfrak{M}, \eta)$ and $\mathfrak{M}_{\rho}=(\mathfrak{M}, \rho)$, and henceforth identify their tangent spaces.

The cover of $\mathfrak{M}_{\rho}$ corresponding to $\partial M$ is a quasifuchsian manifold $\operatorname{qf}\left(Z, \sigma_{M}(Z)\right)$, see Figure 5.

We now show that there is a constant $A_{5}=A_{5}(\chi(S), \operatorname{injrad}(Y)) \geq A_{4}$ such that the Teichmüller distance between $\sigma_{M}(Z)$ and $\bar{Y}$ is less than $2 A_{5} e^{-n}$.

Consider the surface $\mathscr{E}_{n+3}^{2}$ in $\mathfrak{M}_{\eta}$. Let $\mathscr{F}_{n}$ be the image of $\mathscr{E}_{n+3}^{2}$ in $\mathfrak{M}_{\rho}$, see Figure 5. A small neighborhood of $\mathscr{F}_{n}$ lifts isometrically to $\mathrm{qf}\left(Z, \sigma_{M}(Z)\right)$, and we continue to use $\mathscr{F}_{n}$ to denote this lift. Let $\mathbf{n}$ be the field of $\eta$-unit normal vectors to $\mathscr{E}_{n+3}^{2}$ in $\mathfrak{M}_{\eta}$ pointing toward $\bar{Y}$. Let $\mathbf{u}$ be an $\eta$-unit vector field tangent to $\mathscr{E}_{n+3}^{2}$ on some open patch of $\mathscr{E}_{n+3}^{2}$. The normal curvature of $\mathscr{E}_{n+3}^{2}$ along $\mathbf{u}$ is given by

$$
\mathscr{N}_{\mathscr{E}_{n+3}^{2}}(\mathbf{u})=\frac{\mathbf{I}_{\mathscr{E}_{n+3}^{2}}(\mathbf{u}, \mathbf{u})}{\|\mathbf{u}\|_{\eta}^{2}}=-\frac{\eta\left(\mathbf{n}, \nabla_{\mathbf{u}}^{\eta} \mathbf{u}\right)}{\|\mathbf{u}\|_{\eta}^{2}}=-\eta\left(\mathbf{n}, \nabla_{\mathbf{u}}^{\eta} \mathbf{u}\right)
$$

- Note that $T$ does depend on $\operatorname{inj} \operatorname{rad}(Y)$, for we must be at a certain depth in the collar to ensure that the traceless Ricci curvature of our metric vanishes on the thin parts. 


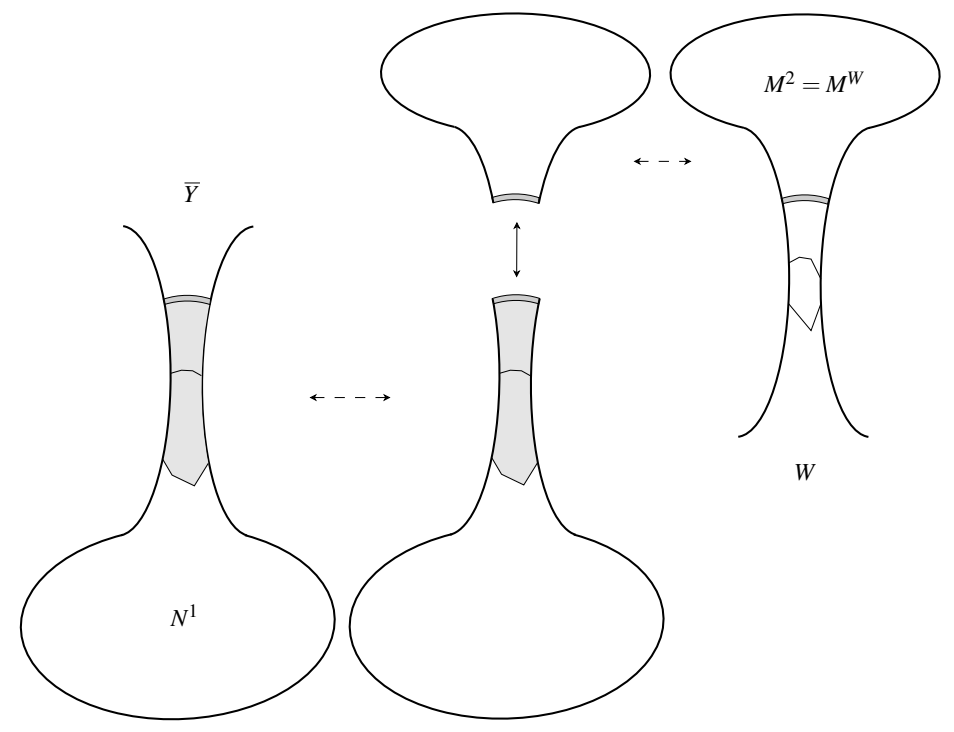

Figure 4: Building $\mathfrak{M}$ with the metric $\eta$.

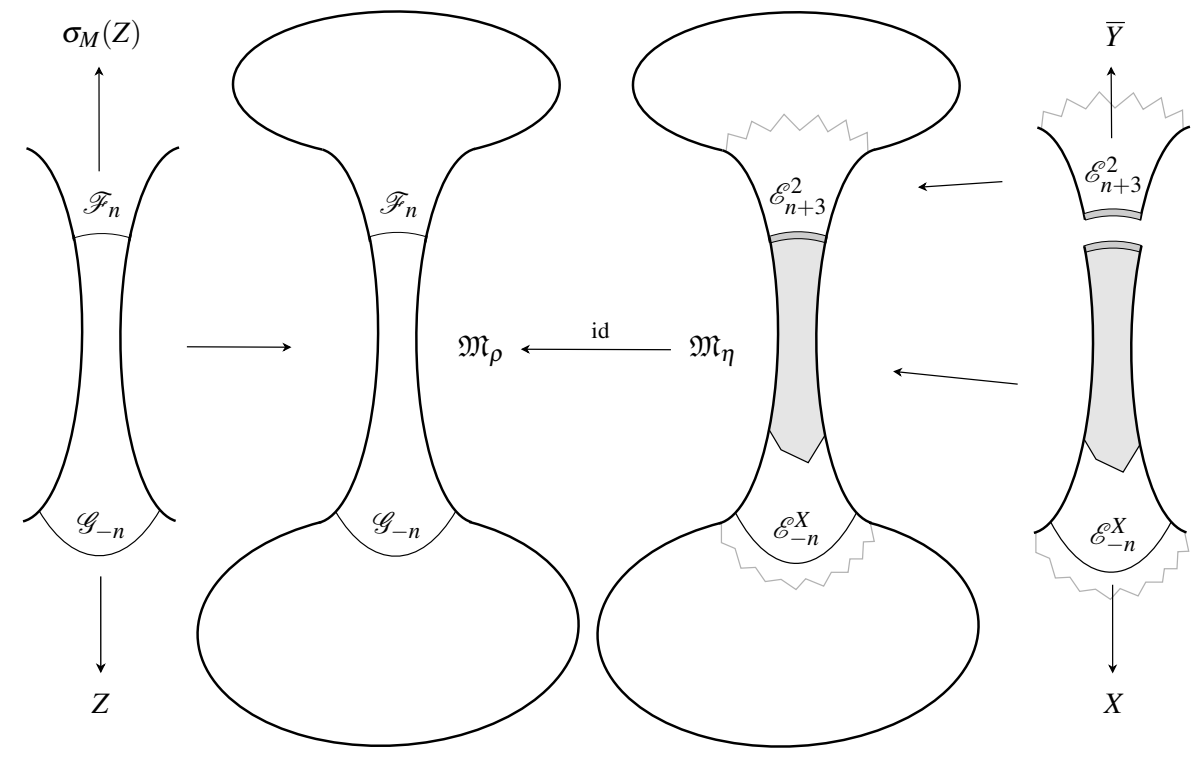

Figure 5: The manifold $\mathfrak{M}$ with its two metrics $\rho$ and $\eta$. At left is the covering map qf $\left(Z, \sigma_{M}(Z)\right) \rightarrow \mathfrak{M}_{\rho}$, together with the projections $\mathscr{G}_{-n} \rightarrow Z$ and $\mathscr{F}_{n} \rightarrow \sigma_{M}(Z)$. At right are partial covering maps from subsets of $\mathrm{qf}(Y, \bar{Y})$ and $\mathrm{qf}(X, \bar{Y})$ to $\mathfrak{M}_{\eta}$ with corresponding projections $\mathscr{E}_{n+3}^{2} \rightarrow \bar{Y}$ and $\mathscr{E}_{-n} X \rightarrow X$. 
where $\nabla^{\eta}$ is the Levi-Civita connection for $\eta$. By Lemma 12, this curvature is within $9 e^{-2 n}$ of 1 .

Letting $\mathbf{m}$ be the $\rho$-unit normal field to $\mathscr{F}_{n}$ pointing toward the skinning surface $\sigma_{M}(Z)$, the normal curvature of $\mathscr{F}_{n}$ is given by

$$
\mathscr{N}_{\mathscr{F}_{n}}(\mathbf{u})=\frac{\mathbb{I}_{\mathscr{F}_{n}}(\mathbf{u}, \mathbf{u})}{\|\mathbf{u}\|_{\rho}^{2}}=-\frac{\rho\left(\mathbf{m}, \nabla_{\mathbf{u}}^{\rho} \mathbf{u}\right)}{\|\mathbf{u}\|_{\rho}^{2}}
$$

where $\nabla^{\rho}$ is the Levi-Civita connection for $\rho$. The proximity (5.1) of the metrics provides the following estimate.

Claim. The normal curvatures satisfy

$$
\left|\mathscr{N}_{\mathscr{E}_{n+3}^{2}}(\mathbf{u})-\mathscr{N}_{\mathscr{F}_{n}}(\mathbf{u})\right|=\mathscr{O}\left(e^{-n}\right) .
$$

Proof of claim. We have $\left\|\nabla_{\mathbf{u}}^{\eta} \mathbf{u}\right\|_{\eta}=\mathscr{O}(1)$, and so (5.1) gives us

$$
\left\|\nabla_{\mathbf{u}}^{\rho} \mathbf{u}\right\|_{\eta}+\left\|\nabla_{\mathbf{u}}^{\rho} \mathbf{u}\right\|_{\rho}=\mathscr{O}(1) .
$$

Together with (5.1), this yields

$$
\left|\eta\left(\mathbf{n}, \nabla_{\mathbf{u}}^{\eta} \mathbf{u}\right)-\rho\left(\mathbf{n}, \nabla_{\mathbf{u}}^{\rho} \mathbf{u}\right)\right|=\mathscr{O}\left(e^{-n}\right) .
$$

Proposition 13 gives us

$$
\|\mathbf{n}-\mathbf{m}\|_{\eta}+\|\mathbf{n}-\mathbf{m}\|_{\rho}=\mathscr{O}\left(e^{-n}\right),
$$

and then (5.5) and the Cauchy-Schwarz inequality give us

$$
\left|\rho\left(\mathbf{n}, \nabla_{\mathbf{u}}^{\rho} \mathbf{u}\right)-\rho\left(\mathbf{m}, \nabla_{\mathbf{u}}^{\rho} \mathbf{u}\right)\right| \leq\|\mathbf{n}-\mathbf{m}\|_{\rho}\left\|\nabla_{\mathbf{u}}^{\rho} \mathbf{u}\right\|_{\rho}=\mathscr{O}\left(e^{-n}\right) .
$$

Now,

$$
\left|\|\mathbf{u}\|_{\rho}^{2}-1\right| \leq A_{4} e^{-n} \leq \frac{1}{2},
$$

by (5.1), and we conclude that

$$
\left|\mathscr{N}_{\mathscr{E}_{n+3}^{2}}(\mathbf{u})-\mathscr{N}_{\mathscr{F}_{n}}(\mathbf{u})\right|=\left|\frac{\eta\left(\mathbf{n}, \nabla_{\mathbf{u}}^{\eta} \mathbf{u}\right)}{\|\mathbf{u}\|_{\eta}^{2}}-\frac{\rho\left(\mathbf{m}, \nabla_{\mathbf{u}}^{\rho} \mathbf{u}\right)}{\|\mathbf{u}\|_{\rho}^{2}}\right|=\mathscr{O}\left(e^{-n}\right)
$$

by the triangle inequality.

Claim

So there is an $A_{5}=A_{5}(\chi(S), \operatorname{injrad}(Y)) \geq A_{4}$ such that the normal curvatures of $\mathscr{F}_{n}$ are within $A_{5} e^{-n}$ of 1 . It follows that there is an $n_{0}=n_{0}(\chi(S), \operatorname{injrad}(Y))$ such that, for $n \geq n_{0}$, the normal projection $v_{n}: \mathscr{F}_{n} \rightarrow \sigma_{M}(Z)$ is defined and nonsingular. Lemma 
11 tells us that $v_{n}$ is $\left(1+A_{5} e^{-n}\right)^{2}$-quasiconformal and that $\mathscr{E}_{n+3}^{2}$ is $\left(1+9 e^{-2 n}\right)^{2}$ quasiconformally equivalent to $\bar{Y}$. Since the map $\mathscr{E}_{n+3}^{2} \rightarrow \mathscr{F}_{n}$ is $\left(1+A_{4} e^{-n}\right)^{2}$-quasiconformal, we conclude that the Teichmüller distance between $\sigma_{M}(Z)$ and $\bar{Y}$ is no more than $\log \left(1+A_{4} e^{-n}\right)+\log \left(1+9 e^{-2 n}\right)+\log \left(1+A_{5} e^{-n}\right) \leq 3 A_{5} e^{-n}$.

We now claim that there is an $A_{6}=A_{6}(\chi(S), \operatorname{injrad}(Y)) \geq A_{5}$ such that $Z$ is within $3 A_{6} e^{-n}$ of $X$. To see this, let $\mathscr{E}_{-n}^{X}$ be the Epstein surface in the $X$-end of $\operatorname{qf}(X, \bar{Y})$ at distance $n$. By our assumption on $X$, a small neighborhood of this surface embeds isometrically in $N^{1}$, and hence $\mathfrak{M}_{\eta}$. We let $\mathscr{G}_{-n}$ denote this surface considered in $\mathfrak{M}_{\rho}$. By (4.2), the principal curvatures of $\mathscr{E}_{-n}^{X}$ are within $9 e^{-2 n}$ of 1 . So the principal curvatures of $\mathscr{G}_{-n}$ are $1+\mathscr{O}\left(e^{-n}\right)$, as in the above argument. By Lemma 11, the projection $v_{-n}: \mathscr{G}_{-n} \rightarrow Z$ is $\left(1+A_{6} e^{-n}\right)^{2}$-quasiconformal for some $A_{6}$. Since proximity of the metrics tells us that $\mathscr{G}_{-n}$ and $\mathscr{E}_{-n}^{X}$ are $\left(1+A_{4} e^{-n}\right)^{2}$-quasiconformal, and $\mathscr{E}_{-n}^{X}$ is $\left(1+e^{-n}\right)^{2}$-quasiconformally equivalent to $X$, we conclude that the Teichmüller distance between $Z$ and $X$ is less than $3 A_{6} e^{-n}$.

By Royden's theorem that the Teichmüller and Kobayashi metrics agree [22], the skinning map is 1-lipschitz, as it is holomorphic. ${ }^{\bullet}$ We conclude that the distance between $\sigma_{M}(X)$ and $\sigma_{M}(Z)$ is at most $3 A_{6} e^{-n}$, and so the distance between $\sigma_{M}(X)$ and $\bar{Y}$ is at most $6 A_{6} e^{-n}$.

Using circle packings with very small circles in the proof of Brooks's theorem [7] (as done in Theorems 31 and 33 of [12]) demonstrates that any Riemann surface $V$ is within $A_{6} e^{-n}$ of an $X$ such that the $2 n$-neighborhood of the convex core of $Q(X, \bar{Y})$ together with the $\bar{Y}$-end of $Q(X, \bar{Y})$ embeds into a convex cocompact hyperbolic 3manifold $N^{1}$ with conformal boundary $\bar{Y}$. Since skinning maps are 1-lipschitz, we conclude that the diameter of $\sigma_{M}$ is no more than $7 A_{6} e^{-n}=7 A_{6} e^{-(\lfloor d\rfloor-8)} \leq 56722 A_{6} e^{-d}$.

Since the metrics $\eta$ and $\rho$ are within $C_{\text {Tian }} A_{0} e^{-n}$ of each other, the images $\mathscr{F}_{t}$ of the $\mathscr{E}_{t}^{2}$ in $\mathfrak{M}_{\rho}$ are convex for $t$ greater than our chosen $T$. It follows that there is a $K_{0}=K_{0}(\chi(S), \operatorname{injrad}(Y))$ such that the $K_{0} d$-neighborhood of the convex core of qf $\left(Z, \sigma_{M}(Z)\right)$ embeds in $M^{Z}$. Since $X$ is within $3 A_{6} e^{-n}$ of $Z$, Corollary B.23 and Proposition 2.16 of [16] ${ }^{\triangle}$ provide a $K_{1}=K_{1}(\chi(S), \operatorname{injrad}(Y))$ such that the $K_{1} d$-neighborhood of the convex core of $\operatorname{qf}\left(X, \sigma_{M}(X)\right)$ embeds in $M^{X}$. As any Riemann surface is within $A_{6} e^{-n}$ of such an $X$, another application of Corollary B.23 and Proposition 2.16 of [16] provide a $K=K(\chi(S), \operatorname{injrad}(Y))$ such that the $K d$-neighborhood of the convex core of qf $\left(V, \sigma_{M}(V)\right)$ embeds in $M^{V}$ for any $V$.

This completes the proof of Theorem 2.

\section{References}

[1] Lars V. Ahlfors. Lectures on quasiconformal mappings. Manuscript prepared with the assistance of Clifford J. Earle, Jr. Van Nostrand Mathematical Studies,

\footnotetext{
$\bullet$ A more direct argument may be found in Section 16.3 of [11]. The argument there establishes the stronger statement that skinning maps are strictly distance-decreasing unless the 3-manifold is an interval bundle over a surface. See [15] for another proof of this, as well as a proof that skinning maps of acylindrical manifolds are uniformly contracting.

${ }^{\triangle}$ Corollary B.23 says that $L$-quasiconformal conjugacies of Kleinian groups provide $L^{3 / 2}$-bilipschitz maps between hyperbolic manifolds, and Proposition 2.16 bounds the distance between convex cores of bilipschitz manifolds.
} 
No. 10. D. Van Nostrand Co., Inc., Toronto, Ont.-New York-London, 1966.

[2] C. Greg Anderson. Projective structures on Riemann surfaces and developing maps to $\mathbb{H}^{3}$ and $\mathbb{C} P^{n}$. University of California at Berkeley PhD thesis, 1998.

[3] Lipman Bers. Simultaneous uniformization. Bull. Amer. Math. Soc., 66:94-97, 1960.

[4] Lipman Bers. On boundaries of Teichmüller spaces and on Kleinian groups. I. Ann. of Math. (2), 91:570-600, 1970.

[5] Lipman Bers. Spaces of Kleinian groups. In Several Complex Variables, I (Proc. Conf., Univ. of Maryland, College Park, Md., 1970), pages 9-34. Springer, Berlin, 1970.

[6] Kenneth W. Bromberg. Hyperbolic cone-manifolds, short geodesics, and Schwarzian derivatives. J. Amer. Math. Soc., 17(4):783-826 (electronic), 2004.

[7] Robert Brooks. Circle packings and co-compact extensions of Kleinian groups. Invent. Math., 86(3):461-469, 1986.

[8] Richard D. Canary and Darryl McCullough. Homotopy equivalences of 3manifolds and deformation theory of Kleinian groups. Mem. Amer. Math. Soc., 172(812):xii+218, 2004.

[9] Charles L. Epstein. Envelopes of horospheres and Weingarten surfaces in hyperbolic 3-space. Preprint, 1984.

[10] Sylvestre Gallot, Dominique Hulin, and Jacques Lafontaine. Riemannian geometry. Universitext. Springer-Verlag, Berlin, third edition, 2004.

[11] Michael Kapovich. Hyperbolic manifolds and discrete groups, volume 183 of Progress in Mathematics. Birkhäuser Boston Inc., Boston, MA, 2001.

[12] Richard Peabody Kent, IV. Skinning maps. Duke Math. J., 151(2):279-336, 2010.

[13] W. Kraus. Über den Zusammenhang einiger Characteristiken eines einfach zusammenhängenden Bereiches mit der Kreisabbildung. Mitt. Math. Sem. Giessen, 21:1-28, 1932.

[14] Albert Marden. The geometry of finitely generated kleinian groups. Ann. of Math. (2), 99:383-462, 1974.

[15] Curtis T. McMullen. Iteration on Teichmüller space. Invent. Math., 99(2):425$454,1990$.

[16] Curtis T. McMullen. Renormalization and 3-manifolds which fiber over the circle, volume 142 of Annals of Mathematics Studies. Princeton University Press, Princeton, NJ, 1996. 
[17] John W. Morgan. On Thurston's uniformization theorem for three-dimensional manifolds. In The Smith conjecture (New York, 1979), volume 112 of Pure Appl. Math., pages 37-125. Academic Press, Orlando, FL, 1984.

[18] David Mumford. A remark on Mahler's compactness theorem. Proc. Amer. Math. Soc., 28:289-294, 1971.

[19] Zeev Nehari. The Schwarzian derivative and schlicht functions. Bull. Amer. Math. Soc., 55:545-551, 1949.

[20] Jean-Pierre Otal. Thurston's hyperbolization of Haken manifolds. In Surveys in differential geometry, Vol. III (Cambridge, MA, 1996), pages 77-194. Int. Press, Boston, MA, 1998.

[21] Jean-Pierre Otal. The hyperbolization theorem for fibered 3-manifolds, volume 7 of SMF/AMS Texts and Monographs. American Mathematical Society, Providence, RI, 2001. Translated from the 1996 French original by Leslie D. Kay.

[22] H. L. Royden. Automorphisms and isometries of Teichmüller space. In Advances in the Theory of Riemann Surfaces (Proc. Conf., Stony Brook, N.Y., 1969), pages 369-383. Ann. of Math. Studies, No. 66. Princeton Univ. Press, Princeton, N.J., 1971.

[23] Dennis Sullivan. Quasiconformal homeomorphisms and dynamics. II. Structural stability implies hyperbolicity for Kleinian groups. Acta Math., 155(3-4):243$260,1985$.

[24] William P. Thurston. Hyperbolic geometry and 3-manifolds. In Low-dimensional topology (Bangor, 1979), volume 48 of London Math. Soc. Lecture Note Ser., pages 9-25. Cambridge Univ. Press, Cambridge, 1982.

[25] Gang Tian. A pinching theorem on manifolds with negative curvature. In Proceedings of International Conference on Algebraic and Analytic Geometry. Tokyo, 1990.

Department of Mathematics, University of Wisconsin - Madison, Madison, Wi 53706

rkent@math. wisc . edu

Department of Mathematics, Yale University, New Haven, CT 06520

yair.minsky@yale.edu 